\title{
Parcours initiatique en phytothérapie
}

Rien ne vaut l'expérience : « Le meilleur médecin est celui qui a eu toutes les maladies " (car il en connaît tous les symptômes).

Le meilleur phytothérapeute est celui qui n'aura pas appris toute la matière sur les bancs de la faculté, et qui n'aura pas uniquement eu pour école le contact avec la nature. Actuellement, les

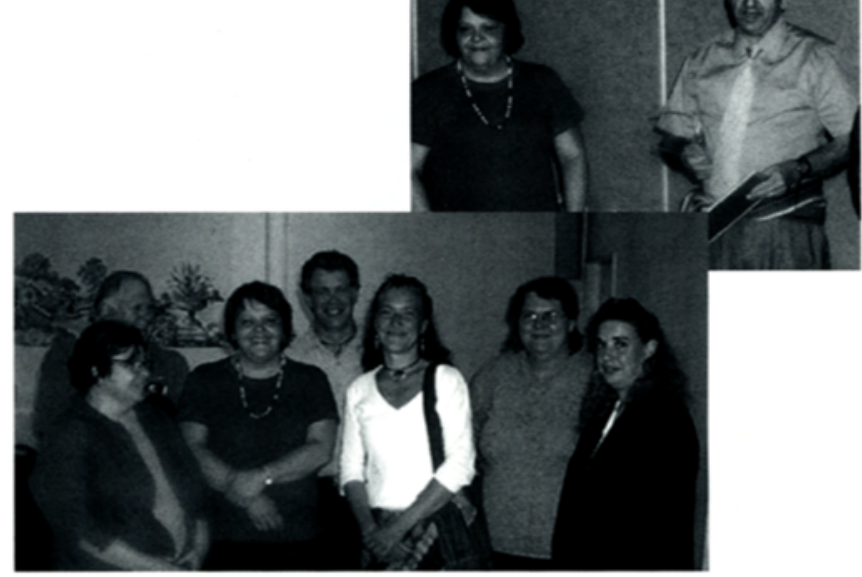
enseignants de phytothérapie réfléchissent au contenu des cours dispensés à la faculté de médecine de Paris-XIII. Car il s'agit d'harmoniser cet enseignement pour que les étudiants aient une vue globale de cette thérapie par les plantes.

Retour aux sources : votre serviteur écrit ces lignes du Canada où, lors d'une conférence, il rencontre la première promotion d'élèves en phytothérapie française par correspondance. Sur le terrain, en compagnie des plantes médicinales d'Amérique du Nord (lédon, cimicifuga, ginseng américain, etc.), il est possible de ressentir cette vision globale de la phytothérapie. Les enseignants de botanique doivent s'évertuer à orienter leurs cours sur l'aspect médicinal des simples. Les enseignants de pharmacologie des plantes doivent reconsidérer l'objectif phytomédicament en fonction de l'effet clinique. Le clinicien se doit de connaître à fond la matière médicale, d'avoir la fibre « bio » de la demande du patient tout en gardant à l'esprit qu'une vraie phytothérapie est une phytothérapie efficace.

Il y a vingt ans, les enseignants de Bobigny disaient devoir faire le ménage et le tri dans le fouillis des publications en cette discipline. Il y a eu beaucoup de progrès depuis, voire trop, dans le sens de la critique de la réalité de l'efficacité de la phytothérapie. Les opposants tiennent un double discours : d'une part, la plante médicinale n'a pas d'effet thérapeutique ou même biologique, d'autre part, elle a des interactions avec les médicaments de synthèse.

Le meilleur témoignage vient de nos patients qui confirment l'efficacité d'une médication ou qui viennent vanter celle d'une automédication à base de plante.

L'initiation en phytothérapie nécessite un plongeon au sein du végétal tel qu'il est, une descente dans la jungle des molécules constituantes des plantes, de leurs effets pharmacologiques complexes, et enfin une confrontation de la réalité de

légende photo : conférence de médecine naturelle à Granby (Canada); élèves de phytothérapie et organisateurs des cours (C. Chartier, P. Goetz) 
l'application de phytothérapie quotidienne de chacun à l'examen de l'evidence based medicine.

C'est là le passage initiatique obligatoire pour être phytothérapeute.

C'est en somme le principe que nous appliquons dans votre revue lors de l'établissement des monographies "médicalisées " qui sont désormais signées par plusieurs auteurs compétents.

$\mathrm{D}^{\mathrm{r}}$ Paul Goetz rédacteur en chef (Granby, province du Québec, juin 2006) 